\title{
Alendronate Associated Minimal Change Disease
}

\author{
Chenna $\mathrm{S}^{1}$, Tindni $\mathrm{A}^{\mathbf{1}}$, Puri $\mathrm{V}^{\mathbf{1}}$ and Chippa $\mathrm{MV}^{2 *}$ \\ ${ }^{1}$ Nephrology associates of Greater Cincinnati, USA \\ ${ }^{2}$ Mercy Health West Hospital, Hospital Medicine, USA \\ *Corresponding author: Chippa MV, Hospital \\ Medicine, Mercy West hospital, 3300 Mercy Health Blvd, \\ Cincinnati, Ohio, USA
}

Received: December 24, 2018; Accepted: J anuary 30, 2019; Published: February 06, 2019

\section{(Austin Publishing rroup}

\begin{abstract}
Kidney injury associated with bisphosphonates is well known in literature, though it is more common with intravenous bisphosphonates. Case reports about focal segmental glomerulosclerosis are reported with oral and intravenous bisphosphonates. We present a case of 87-year-old female with acute renal failure and volume overload found to have minimal change disease on biopsy while on Alendronate for a period of four months. She has failed oral prednisone treatment and is now dependent on hemodialysis. This is probably the first case report of minimal change disease with oral bisphosphonates.
\end{abstract}

Keywords: Alendronate; Minimal change disease; Hemodialysis; Acute renal failure

\section{Abbreviations}

MM: Milli Meters; HG: Mercury; g/dl: Grams Per Deciliter; LPF: Low Power Field; WBC: White Blood Cell; HPF: High Power Field; RBC: Red Blood Cell; HIV: Human Immunodeficiency Virus; ANA: Anti Nuclear Antibody; pANCA: perinuclear Anti Neutrophil Cytoplasmic Antibody; GBM: Glomerular Basement Membrane; Ab: Antibody; PO: Per Oral

\section{Introduction}

Bisphosphonates related kidney disease is well documented in various clinical reports $[1,2]$. Its commonly seen with intravenous Pamidronate, and the most common kidney pathology is focal segmental glomerulosclerosis and less commonly acute tubular necrosis $[1,3,4]$. The clinical course is unpredictable $[2,5]$. Here we present a case of 87-year-old female who was on oral Alendronate and no known kidney disease presenting with progressive shortness of breath and weight gain for 3-4 weeks and was found to be in severe acute renal failure and her kidney biopsy showed minimal change disease and acute tubular necrosis. She was started on high dose prednisone and hemodialysis for uremia. This case suggests, the importance of monitoring kidney function and proteinuria in patients who are on bisphosphonates.

\section{Case Presentation}

An 87-year-old female with past medical history of hypertension, diabetes mellitus type 2 , hyperlipidemia, asthma and hypothyroidism presented to emergency room for the evaluation of progressive swelling in legs associated with shortness of breath and weight gain over 3-4 weeks. At presentation, her blood pressure was 150/68 $\mathrm{mm}$ of $\mathrm{Hg}$, and her physical examination showed diffuse anasarca, distended neck veins and crackles in both the lungs. Her blood urea nitrogen/ serum creatinine was $92 / 3.7$ compared to 26/1.2 a month ago. Her serum albumin was $4 \mathrm{~g} / \mathrm{dl}$ and Urinalysis showed 3 casts/lpf, $303 \mathrm{wbc} /$ hpf, negative blood, $2 \mathrm{rbc} / \mathrm{hpf}$, no glucose. Urine protein to creatinine ratio was $4.79 \mathrm{mg} / \mathrm{g}$. Previous urinalysis was negative for proteinuria. Her creatinine continued to worsen and reached to a peak of $7.7 \mathrm{mg} /$ $\mathrm{dl}$ in 48 hours. A complete workup for nephrotic syndrome was done. She was tested negative for HIV, hepatitis B and C. Her autoimmune work including complement levels, ANA and pANCA were normal. Anti GBM Ab was negative as well.

She underwent left kidney biopsy, it had 38 glomeruli. It showed minimal change disease, global glomerulosclerosis with acute tubular necrosis and interstitial fibrosis (Figures $1 \& 2$ ).

The medications she was taking at home included Alendronate, Bupropion, Sitagliptin, Lovastatin, Metformin, MiraLAX, Losartan, Calcium and Vit D. She denied use of over the counter Non-steroidal anti-inflammatory drugs, herbal medications. In the absence of other causes for nephrotic syndrome and recent use of Alendronate (started about 4 months ago), we strongly suspect the possible relationship of minimal change disease to oral Alendronate, given bisphosphonates are associated with nephrotic syndrome.

With rapidly worsening uremia, pulmonary edema and no response to intravenous diuretics, on day 2 of admission she had to be started on hemodialysis. She was started on oral prednisone 60 mg po daily. Based on biopsy results she was continued on high dose prednisone. She did not respond to steroids over the next two months and continued to be dialysis dependent.

\section{Discussion}

Bisphosphonates are commonly used for the treatment of osteoporosis in men and postmenopausal women [6], prevention of osteoporosis in postmenopausal women, treatment of glucocorticoid-

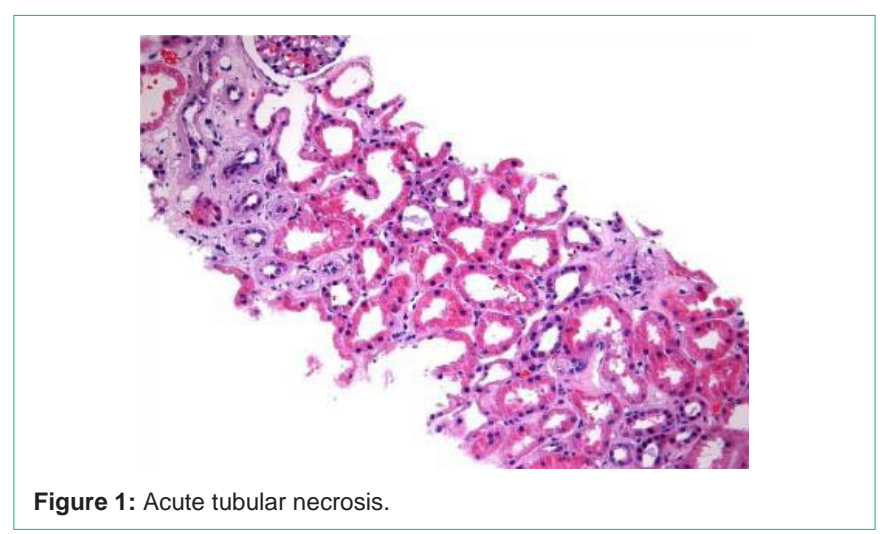




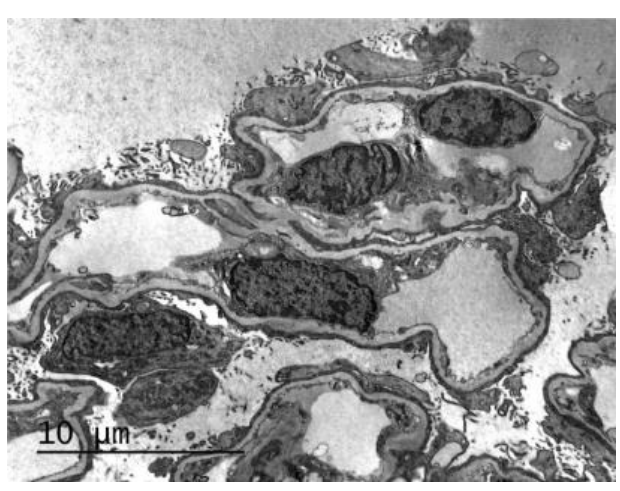

Figure 2: Extensive foot process effacement.

induced osteoporosis [7], treatment of Paget's disease of bone [8], multiple myeloma with lytic bone lesions and severe osteoporosis $[9,10]$, and in breast cancer with bone metastasis [11]. There are multiple case reports of focal segmental glomerulosclerosis with and without acute renal failure related to intravenous pamidronate $[1,12,13]$ as well of oral bisphosphonates [2].

In review of literature pertaining to focal segmental glomerulosclerosis with Alendronate there are five reported cases, of them two cases had complete remission, one being 36-year-old male with osteoporosis, who was in remission with just stopping Alendronate [5] and other 61-year-old Japanese male on oral Alendronate use for just 14 days developed severe proteinuria and acute renal failure, got better on steroid and stopping Alendronate and requiring six cycles of hemodialysis and four cycles of LDL apheresis [2], and the other three had partial remission.

Another oral bisphosphonate Etidronate was associated with recurrence of minimal change disease with complete remission with oral steroids and withdrawal of bisphosphonate [14]. Our patient developed minimal change disease after taking Alendronate for four months. This is probably the first case report of an association between minimal change disease and alendronate. As previously published reports, the course of nephrotic syndrome with oral bisphosphonates is so varied, and our patient ended up on hemodialysis and had no response to oral steroids and stopping Alendronate.

The bisphosphonates act by inhibiting osteoclastic induced bone resorption, decreasing osteoclast progenitor development, recruitment and promotion of osteoclast apoptosis. In addition, they also appear to have a beneficial effect on osteoblasts. The exact mechanism of renal injury from bisphosphonates is not very clear, but the most common accepted hypothesis is the injury to podocyte cytoskeleton structure which is similar an osteoclast. Nephrotic syndrome from bisphosphonates can be seen in patients who are on it for a long time [3] as well as very short time, even with single intravenous dose [15], our patient was on Alendronate for a period of 4 months. In general, elderly people will have poor outcome compared to young people $[2,5]$.

Nephrotic syndrome is reported with both intravenous and oral bisphosphonates, and the clinical course is unpredictable which may even lead to permanent hemodialysis. Bisphosphonates have wide variety of indications and the risk of renal failure in elderly population is life changing especially if requires permanent hemodialysis. There is no known prevention of these complications and caution should be used in patients with chronic kidney disease [16]. Routine use of urine dipstick may be of value. When prescribing oral bisphosphonates, we should keep in mind these rare but significant adverse complications. In these particular situations, we will have to find alternative treatment options for osteoporosis.

\section{References}

1. Markowitz GS, Appel GB, Fine PL, Fenves AZ, Loon NR, Jagannath S, et al. Collapsing focal segmental glomerulosclerosis following treatment with highdose pamidronate. J Am Soc Nephrol. 2001; 12: 1164-1172.

2. Miura N, Mizuno N, Aoyama R, Kitagawa W, Yamada H, Nishikawa K, et al. Massive proteinuria and acute renal failure after oral bisphosphonate (alendronate) administration in a patient with focal segmental glomerulosclerosis. Clin Exp Nephrol. 2009; 13: 85-88.

3. Garimella PS, Rennke HG, Strom JA. Alendronate associated focal segmental glomerulosclerosis: a case report and review of the literature. CEN Case Rep. 2015; 4: 20-23.

4. Barri YM, Munshi NC, Sukumalchantra S, Abulezz SR, Bonsib SM, Wallach J, et al. Podocyte injury associated glomerulopathies induced by pamidronate. Kidney Int. 2004; 65: 634-641.

5. Yilmaz M, Taninmis H, Kara E, Ozagari A, Unsal A. Nephrotic syndrome after oral bisphosphonate (alendronate) administration in a patient with osteoporosis. Osteoporosis Int. Osteoporos Int. 2012; 23: 2059-2062.

6. Fleisch H. Bisphosphonates in osteoporosis. Eur Spine J. 2003; 12: S142-S146.

7. Recommendations for the prevention and treatment of glucocorticoid-induced osteoporosis: 2001 update. American College of Rheumatology Ad Hoc Committee on Glucocorticoid-Induced Osteoporosis. Arthritis Rheum. 2001; 44: 1496-1503.

8. Singer FR. Paget disease: When to treat and when not to treat. Nat Rev Rheumatol. 2009; 5: 483-489.

9. Samantha Pozzi, Noopur Raje. The Role of Bisphosphonates in Multiple Myeloma: Mechanisms, Side Effects, and the Future. Oncologist. 2011; 16 : 651-662.

10. Terpos E, Sezer O, Croucher PI, García-Sanz R, Boccadoro M, San Migue $\mathrm{J}$, et al. The use of bisphosphonates in multiple myeloma: Recommendations of an expert panel on behalf of the European Myeloma Network. Ann Oncol. Ann Oncol. 2009; 20: 1303-1317.

11. Pavlakis N, Stockler M. Bisphosphonates for breast cancer. Cochrane Database Syst Rev. 2002; CD003474.

12. Perazella MA, Markowitz GS. Bisphosphonate nephrotoxicity. Kidney Int. 2008; 74: 1385-1393.

13. Sauter M, Jülg B, Porubsky S, Cohen C, Fischereder M, Sitter T, et al. Nephrotic-range proteinuria following pamidronate therapy in a patient with metastatic breast cancer: mitochondrial toxicity as a pathogenetic concept? Am J Kidney Dis. Am J Kidney Dis. 2006; 47: 1075-1080.

14. Stratton JD, Warwicker P. Relapse of nephrotic syndrome triggered by bisphosphonates. Nephrol Dial Transplant. 2002; 17: 1856-1857.

15. Javier AN, Omkar UV, Hendricks A, Sambandam KK. Collapsing Focal Segmental Glomerulosclerosis Resulting from a Single Dose of Zoledronate. Nephron Extra. 2014; 4: 168-174.

16. Pascual J, Torrealba J, Myers J, Tome S, Samaniego M, Musat A, Djamali A. Collapsing focal segmental glomerulosclerosis in a liver transplant recipient on alendronate. Osteoporos Int. 2007; 18: 1435-1438. 